\title{
Continuous Heliox Breathing and the Extent of Anatomic Zone of No- reflow and Necrosis Following Ischemia/Reperfusion in the Rabbit Heart
}

\author{
Sharon L. Hale*,1, Donald R. VanDeripe, and Robert A. Kloner ${ }^{1,2}$, \\ ${ }^{1}$ The Heart Institute of Good Samaritan Hospital, Los Angeles, CA, USA \\ ${ }^{2}$ Keck School of Medicine, Division of Cardiovascular Medicine, University of Southern California, Los Angeles, CA, \\ USA
}

\begin{abstract}
Background: Nitrogen may contribute to reperfusion injury. Some studies have shown that helium as a replacement for nitrogen in breathing gas (heliox) reduces cell necrosis after ischemia/reperfusion when used in a preconditioning fashion (intermittent heliox exposure). Our aim was to test whether heliox, breathed continuously throughout the ischemic and reperfusion periods, reduced necrosis and a marker of reperfusion injury, the no-reflow phenomenon.
\end{abstract}

Methods and Results: Anesthetized, open-chest rabbits received 30 min coronary artery occlusion/3 hrs reperfusion. Before CAO rabbits were randomized to heliox ( $30 \%$ oxygen $+70 \%$ helium, $n=8$ ) or air supplemented with oxygen to achieve blood gas values within physiologic range $(n=8)$. Rabbits received the appropriate mix during ischemic and reperfusion periods. Infarct size (\% risk zone) and no-reflow defect were measured at the end of the reperfusion period. The ischemic risk zone was similar in both groups (28\% of left ventricle in heliox and $29 \%$ in control). Heliox breathing did not reduce necrosis; infarct size, expressed as a percentage of the risk region was $44 \pm 4 \%$ in the heliox group and $49 \pm 5 \%$ in controls, $\mathrm{p}=0.68$. The extent of the no-reflow defect was not altered by heliox, either expressed as a percent of the risk region ( $29 \pm 4 \%$ in heliox and $28 \pm 3 \%$ in control) or as a percent of the necrotic zone ( $65 \pm 5 \%$ in heliox and $59 \pm 8 \%$ in control).Heliox treatment had no effect on hemodynamic parameters or arterial blood gas values.

Conclusion: Continuous heliox breathing does not appear to be cardioprotective in the setting of acute myocardial infarction in the rabbit model. Heliox respiration administered during 30 minutes of ischemia and 180 minutes of reperfusion did not alter infarct size or the extent of no-reflow.

Key words: heliox, no-reflow phenomenon, infarct size, rabbit

\section{INTRODUCTION}

Exposure to heliox, in lieu of nitrogen-containing breathing gas, has been shown to have neuro-protective [1-3] and cardio-protective [4-7] effects in the setting of ischemia/ reperfusion. Suggested mechanisms for these beneficial effects include activation of mitochondrial $\mathrm{Ca}++$ sensitive potassium channels [8], protective kinase-signaling cascades [7] and mild uncoupling leading to decreased ROS (reactive oxygen species) production [4]. It has also been suggested that substituting helium for nitrogen in breathing gas might limit reperfusion injury by a mechanical modus; i.e. reducing nitrogen bubble formation within the cell. Reperfusion injury is a consequence of restoring blood flow to tissue after a period of ischemia. During ischemia, nitrogen is present in the cell in larger amounts than oxygen. Damaged mitochondrial membranes allow nitrogen uptake causing swelling in the mitochondria, making the mitochondria unable to take up

*Address correspondence to this author at the The Heart Institute, Good Samaritan Hospital, 1225 Wilshire Blvd, Los Angeles, CA, 90017 USA;

Tel: 213-977-4045; Fax: 213-977-4107; Email: sharon.hale@netscape.com oxygen when it is restored, limiting recovery of oxidative metabolism and leading to irreversible injury [9].

A reduction in necrosis occurring with ischemia is the primary objective for interventions applied to patients after acute myocardial infarction. However, another key objective is reducing reperfusion injury. The no-reflow phenomenon is an important component of reperfusion injury following infarction and reperfusion and is characterized by an anatomic area of hypo-perfusion or no perfusion related to microvascular obstruction. No-reflow occurs after opening the occluded major epicardial coronary artery. In patients, it is seen using angiography, intracoronary contrast echocardiography or magnetic resonance imaging. Experimentally, it is delineated by vascular tracers such as thioflavin $\mathrm{S}$ and carbon black. Early after the reperfusion of an epicardial coronary artery, zones demonstrating a perfusion defect are small; however over time there is a progressive increase in no-reflow in areas that initially were perfused. In patients, large zones of no-reflow are associated with adverse outcome [10-12]. 
Previous studies in rabbits [7, 13-17] have tested the effects of heliox on necrosis when administered as a preconditioning mimetic and observed a reduction in infarct size. However, a preconditioning intervention has limited clinical application, so the purpose of this study was to test the effects of continuous heliox breathing, starting before the onset of ischemia and continuing throughout reperfusion, on the development of necrosis and no-reflow defect after myocardial ischemia and reperfusion in the rabbit model.

\section{METHODS}

The animals used in these studies were maintained in accordance with the policies and guidelines of the Position of the American Heart Association on research animal use (American Heart Association, 1985) and the Guide for Care and Use of Laboratory Animals (2010). The protocol was approved by The Institutional Animal Care and Use Committee of Good Samaritan Hospital.

\section{Surgical and Experimental Procedures}

The techniques used for the rabbit model of acute myocardial infarction have been described previously [18]. In brief, anesthetized, open-chest male New Zealand White rabbits (2.5 to $3.3 \mathrm{~kg}$ ) received 30 minutes of coronary artery occlusion (CAO) followed by 3 hours of reperfusion. Body temperature was maintained using a heating pad. Arterial blood pressure and heart rate were recorded throughout the procedure. The anatomic no-reflow zone was measured using thioflavin S staining, a fluorescent yellow dye that stains endothelium and serves as a marker of regional perfusion. The no-reflow zone appears as a non-fluorescent, dark area, and regions of perfusion appear brightly fluorescent under ultraviolet light. Blue pigment injected through a catheter in the left atrial appendage with the coronary artery re-occluded demarcated the ischemic risk zone, and necrosis was measured using in vitro staining with triphenyltetrazolium chloride. Measurements of risk zone, no-reflow zone and infarct size were calculated as previously described [18].

\section{Treatment Groups}

During surgical preparation, all rabbits were intubated and respired with room air supplemented with $0.1 \mathrm{~L} / \mathrm{min}$ of $100 \%$ oxygen. The respirator was set to an initial volume of $7 \mathrm{ml} / \mathrm{kg} /$ inspiration and a rate of 55-60 breathes per minute. The volume per inspiration was adjusted to maintain $\mathrm{pO}^{2}$ of $\sim 100 \mathrm{~mm} \mathrm{Hg}$, and the rate was adjusted to maintain $\mathrm{pCO}^{2}$ values between $35-45 \mathrm{~mm} \mathrm{Hg}$. After surgical preparation was completed, the rabbits were randomly assigned to treatment group. If the rabbit was assigned to the control group, no changes to respiration were made. If the rabbit was assigned to the heliox group, the gas mixture was changed to heliox (30\% oxygen and $70 \%$ helium) for the duration of the study (15 min stabilization, $30 \mathrm{~min}$ coronary artery occlusion, and 3 hrs reperfusion). Prospective exclusion criteria were 1) death occurring before the study was completed, or 2) an ischemic risk zone of $<10 \%$ of the left ventricle.

\section{Statistical Analyses}

Data were calculated and tabulated using Excel work sheets. All data summary and statistical analyses were performed using SAS (Version 9.3, Cary, NC). Left ventricular weight, infarct size, area at risk, and area of noreflow were compared using Student's t test. Changes in hemodynamic and arterial blood gas variables over time were analyzed by repeated measures analysis of variance. Analysis of covariance was used to test for a group effect on the regression models of necrotic myocardium with risk zone and no-reflow zone with risk zone and necrosis. Data are expressed as mean \pm SEM.

\section{RESULTS}

\section{Experimental Animals}

Nineteen rabbits were assigned to the study. One control rabbit died of ventricular fibrillation at 8 minutes after coronary artery occlusion. Two hearts (one control and one heliox) were excluded on the basis of the prospective exclusion criterion of an ischemic risk zone $<10 \%$ of the left ventricle. Data here are reported on the remaining 8 rabbits in each group. Rectal temperatures on average remained within $0.2^{\circ} \mathrm{C}$ of baseline $\left(39.0^{\circ} \mathrm{C}\right.$ ) in both groups (data not shown).

\section{Arterial Blood Gas Levels}

Arterial blood gas levels were measured throughout the protocol and used to maintain blood gas levels within physiologic range. There were no differences between groups in $\mathrm{pH}, \mathrm{pCO}^{2}$, $\mathrm{pO} 2$ or oxygen saturation between groups or over the time period of the study (data not shown).

\section{Heart Rate and Blood Pressure}

Both groups were similar with respect to baseline values of heart rate and mean arterial blood pressure (Fig. 1). Heart rates remained stable throughout the duration of the study, but arterial pressures decreased over time after coronary artery occlusion as is normally seen in this model. There were no significant differences between groups. Thus breathing helium instead of nitrogen had a neutral effect on hemodynamics.

\section{Extent of the Ischemic Risk Zone, Necrosis and No- reflow}

The average size of the risk zones, expressed as a percentage of the left ventricle (LV), was similar in both groups and comprised $29 \pm 2 \%$ of the left ventricle in the control group and $28 \pm 2 \%$ in the heliox group (Table 1). Therefore, on average, both groups had an equal size ischemic insult during coronary artery occlusion. Breathing a helium mix instead of a gas containing nitrogen failed to reduce myocardial necrosis. Infarct size, expressed as a percent of the risk region was $49 \pm 5 \%$ in controls and $44 \pm$ $4 \%$ in the heliox group ( $\mathrm{p}=\mathrm{ns} ;$ Fig. 2 ). In addition, the extent of no-reflow was not reduced by heliox treatment, as the no-reflow zone expressed both as a percentage of the risk zone and percentage of the necrotic zone was similar in both groups (Table 1 and Fig. 3 ). 


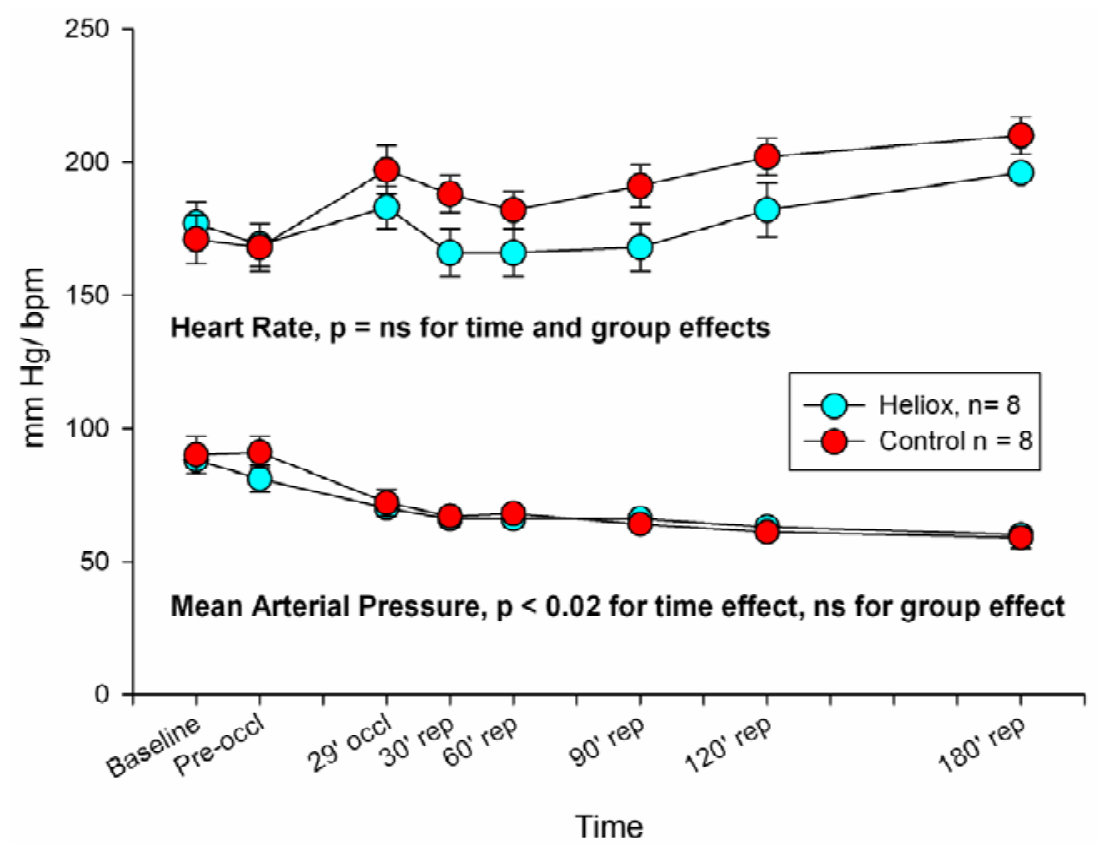

Fig. (1). Heart rate (beats per minute) and mean arterial pressure (mm Hg) in the two groups measured over the study. Heart rates remained stable throughout the duration of the study, but arterial pressures decreased over time after coronary artery occlusion as is normally seen in this model. There were no significant differences between groups.

Ischemic Risk Zone (\%LV)

and Infarct Size (\% Risk Zone)

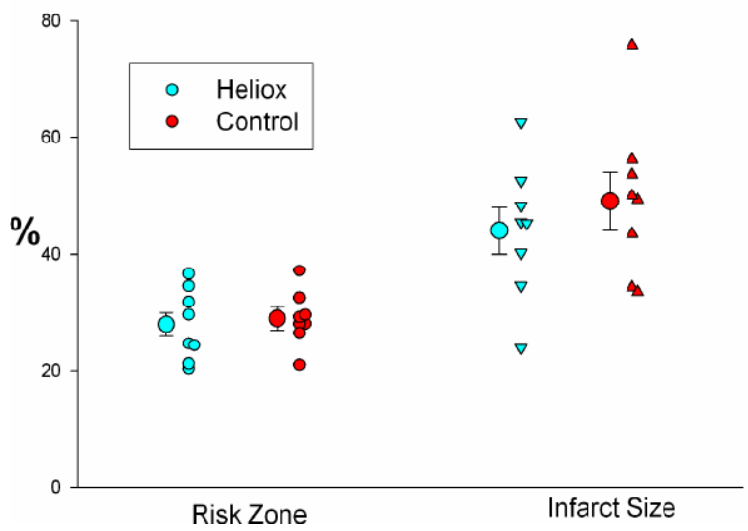

Fig. (2). The average size of the risk zones, expressed as a percentage of the left ventricle (left) was similar in both groups. Therefore, on average, both groups had an equal size ischemic insult during coronary artery occlusion. There was no significant difference between groups in infarct size, expressed as a percentage of the risk zone (right).

\section{DISCUSSION}

\section{Helium and Cardioprotection}

An early study suggested that breathing a helium mixture versus air alone during acute myocardial infarction might be protective. In the late 1960s, Pifarre and coworkers, in one of the first studies examining the potential positive effects of breathing helium, showed that when dogs in the acute phase of circumflex coronary artery occlusion breathed a gas mixture containing 50 percent air, 30 percent oxygen and 20 percent helium, ventricular fibrillation was completely prevented. In another group breathing $20 \%$ oxygen and $80 \%$ helium, VF occurred in $8 \%$; in the control group breathing air, VF occurred in more than half of the animals [19].
It was not until recently that the effects of heliox breathing were tested on the development of myocardial necrosis. In 2007, Pagel and coworkers ${ }^{13}$ tested heliox breathing administered as a preconditioning agent. Rabbits were respired with heliox in 35 -minute cycles interspersed with 5 minutes nitrogen-oxygen mix before index ischemia and reperfusion (30 minutes, followed by three hours of reperfusion). Heliox was not given during the prolongedischemia or reperfusion periods [13]. The authors found that this procedure reduced myocardial infarct size to a degree similar to that of ischemic preconditioning. Data showed that this protection was associated with an activation of prosurvival kinases and inhibition of opening of the mitochondrial permeability transition pore [13]. It has since 


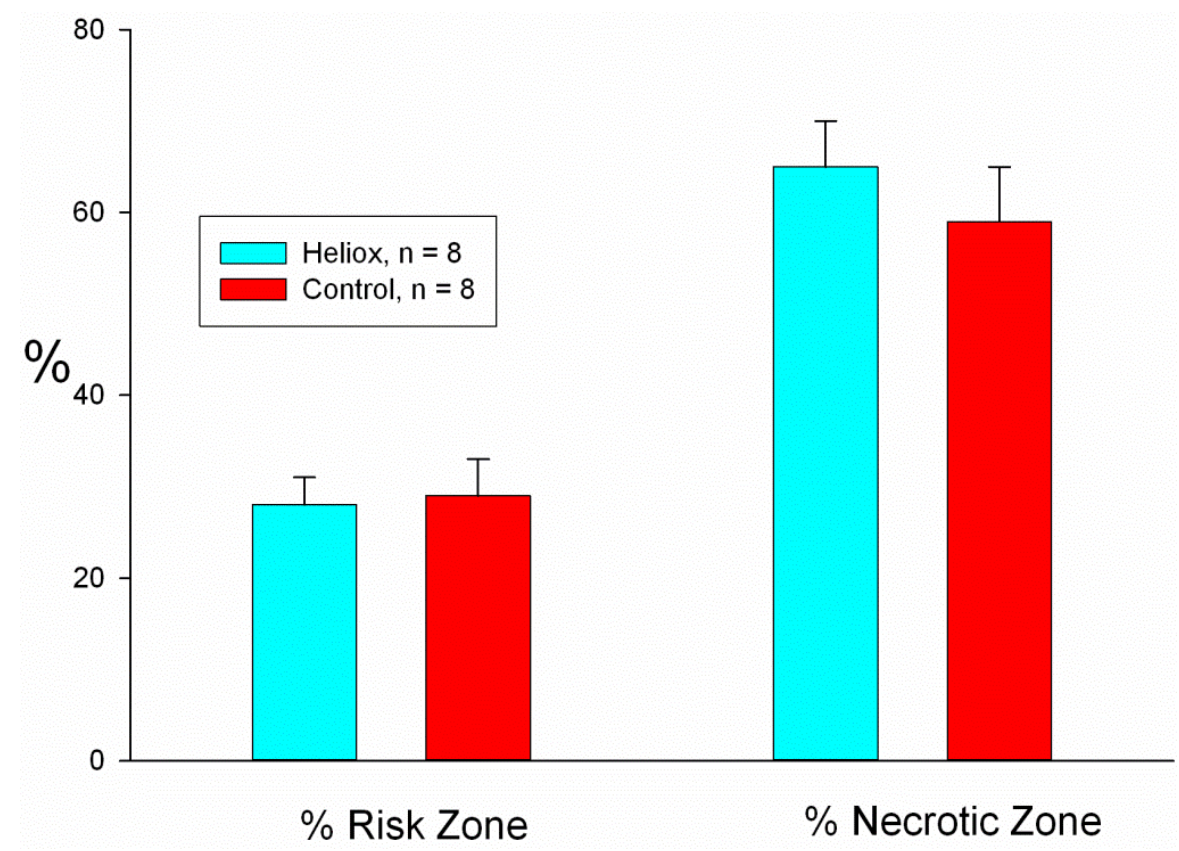

Fig. (3). The no-reflow zone expressed both as a percentage of the risk zone (left) and percentage of the necrotic zone (right) was similar in both groups.

Table 1. Body weight, Risk Region, Infarct Size and No-Reflow in the two treatment groups.

\begin{tabular}{|c|c|c|c|}
\hline Parameter & Control & Heliox & P value \\
\hline \hline Body Weight (Kg) & $2.82 \pm 0.1$ & $2.80 \pm 0.12$ & $3.83 \pm 0.22$ \\
\hline Left Ventricle (LV) Weight (g) & $4.09 \pm 0.25$ & $28 \pm 2$ & 0.45 \\
\hline Risk Region (\%LV) & $29 \pm 2$ & $44 \pm 4$ & 0.68 \\
\hline Infarct Size (\% risk region) & $49 \pm 5 \pm 3$ & 0.41 \\
\hline No-reflow (\% risk region) & $29 \pm 4$ & $65 \pm 5$ & 0.47 \\
\hline No-reflow (\% necrotic region) & $59 \pm 8$ & & 28 \\
\hline
\end{tabular}

been shown that in both rabbits [7, 13, 15] and young (but not old) rats [20], infarct size is reduced by this treatment. In addition, heliox has been shown to be effective in a model of "late preconditioning" [21].

One small clinical study of forearm ischemia tested heliox administered starting 15 minutes before ischemia (15 minutes) and continuing into the reperfusion period. Heliox breathing reduced markers of inflammation, but failed to improve the hyperemic response (author's endpoint for nitric oxide bioavailability and endothelial function) [22].

\section{Results From Our Study}

Although heliox breathing appears to be of benefit when used as a preconditioning agent, preconditioning has limited clinical application for the treatment of acute myocardial infarction. The onset of infarction is unpredictable, and a preconditioning therapy needs to be instituted prior to the onset of myocardial ischemia. Therefore, we chose to test the effect of heliox when administered not in a preconditioning fashion, but throughout ischemia and reperfusion. We tested continuous heliox breathing on infarct size and the extent of no-reflow in our rabbit model. Data from the study indicates that when helium replaces nitrogen in breathing gas during the entire period of ischemia/reperfusion, the heart is not protected from injury. Infarct size and the extent of the noreflow zone were of similar size in both groups. Breathing heliox had a neutral effect on heart rate and blood pressure.

Results from our study are similar to those of an investigation by Fukuda and coworkers [23] who tested the effects of heliox breathing in an acute mouse model of hepatic ischemia/reperfusion. Heliox was started during the ischemic period and continued throughout reperfusion. After 90 minutes of ischemia and 3 hours of reperfusion, cell death, enzyme release and levels of hepatic malondialdehyde were similar in control and heliox breathing animals.

Regarding the effect of heliox on the infarct size there would appear to be a slight, but not statistical, trend for a reduction, and the extremes (20\% on one heliox and $75 \%$ on one control) would tend to support that possibility. A most favorable effect from heliox would be to reduce reperfusion injury following interventions such as TPA, anti-platelet therapy, angioplasty, stent or even by-pass operations. This raises the speculation that heliox might have shown more 
diverging benefit if the rabbits received other treatment, e.g. anti-platelet agents, at the time of ligature release.

\section{Study Limitations}

We did not test heliox breathing as a preconditioning agent to reduce infarct size, nor did we assess the effects of this intervention on myocardial function. Thus we cannot address if heliox would have had a beneficial effect in our model had these been tested. Also, we cannot rule out the possibility that heliox might be beneficial in other cardiac or non-cardiac models of ischemia/reperfusion.

\section{Summary}

Continuous heliox breathing does not appear to be protective in the setting of acute myocardial infarction in the rabbit model. Heliox respiration (30\% oxygen $+70 \%$ helium) given during 30 minutes of ischemia and 180 minutes of reperfusion did not alter infarct size or the extent of no-reflow. Heliox did not change heart rate or blood pressure or arterial blood gas variables.

\section{CONFLICTS OF INTEREST}

The authors confirm that this article content has no conflicts of interest.

\section{ACKNOWLEDGEMENT}

The authors disclose that this study was partially funded by a grant from Donald R. VanDeripe.

\section{REFERENCES}

[1] Liu Y, Xue F, Liu G, et al. Helium preconditioning attenuates hypoxia/ischemia-induced injury in the developing brain. Brain Res 2011; 1376: 122-9.

[2] Pan Y, Zhang H, VanDeripe DR, Cruz-Flores S, Panneton WM. Heliox and oxygen reduce infarct volume in a rat model of focal ischemia. Exp Neurol 2007; 205: 587-90.

[3] Pan Y, Zhang H, Acharya AB, Cruz-Flores S, Panneton WM. The effect of heliox treatment in a rat model of focal transient cerebral ischemia. Neurosci Lett 2011; 497: 144-7.

[4] Huhn R, Heinen A, Weber NC, et al. Helium-induced early preconditioning and postconditioning are abolished in obese Zucker rats in vivo. J Pharmacol Exp Ther 2009; 329: 600-7.

[5] Huhn R, Heinen A, Weber Nc, et al. Helium-induced late preconditioning in the rat heart in vivo. Br J Anaesth 2009; 102: 614-9.

[6] Oei GT, Huhn R, Heinen A, et al. Helium-induced cardioprotection of healthy and hypertensive rat myocardium in vivo. Eur $\mathrm{J}$ Pharmacol 2012; 684: 125-31.

[7] Pagel PS, Krolikowski JG, Pratt PF, et al. The mechanism of helium-induced preconditioning: a direct role for nitric oxide in rabbits. Anesth Analg 2008; 107: 762-8.
[8] Heinen A, Huhn R, Smeele KM, et al. Helium-induced preconditioning in young and old rat heart: impact of mitochondrial $\mathrm{Ca}(2+)$-sensitive potassium channel activation. Anesthesiology 2008; 109: 830-6.

[9] VanDeripe DR. The swelling of mitochondria from nitrogen gas; a possible cause of reperfusion damage. Med Hypotheses 2004; 62: 294-6.

[10] Ito H. No-reflow phenomenon in patients with acute myocardial infarction: its pathophysiology and clinical implications. Acta Med Okayama 2009; 63: 161-8.

[11] Bolognese L, Falsini G, Liistro F, Angioli P, Ducci K. Epicardial and microvascular reperfusion with primary percutaneous coronary intervention. Ital Heart J 2005; 6: 447-52.

[12] Wu KC, Zerhouni EA, Judd RM, et al. Prognostic significance of microvascular obstruction by magnetic resonance imaging in patients with acute myocardial infarction. Circulation 1998; 97: 765-72.

[13] Pagel PS, Krolikowski JG, Shim YH, et al. Noble gases without anesthetic properties protect myocardium against infarction by activating prosurvival signaling kinases and inhibiting mitochondrial permeability transition in vivo. Anesth Analg 2007; 105: 562-9.

[14] Pagel PS, Krolikowski JG, Pratt PF, Jr., Shim YH, Amour J, Warltier DC, Weihrauch D. Inhibition of glycogen synthase kinase or the apoptotic protein p53 lowers the threshold of helium cardioprotection in vivo: the role of mitochondrial permeability transition. Anesth Analg 2008; 107: 769-75.

[15] Pagel PS, Krolikowski JG, Pratt PF, et al. Reactive oxygen species and mitochondrial adenosine triphosphate-regulated potassium channels mediate helium-induced preconditioning against myocardial infarction in vivo. J Cardiothorac Vasc Anesth 2008; 22: 554-9.

[16] Pagel PS, Krolikowski JG. Transient metabolic alkalosis during early reperfusion abolishes helium preconditioning against myocardial infarction: restoration of cardioprotection by cyclosporin A in rabbits. Anesth Analg 2009; 108: 1076-82.

[17] Pagel PS, Krolikowski JG, Amour J, Warltier DC, Weihrauch D. Morphine reduces the threshold of helium preconditioning against myocardial infarction: the role of opioid receptors in rabbits. J Cardiothorac Vasc Anesth 2009; 23: 619-24.

[18] Hale SL, Kloner RA. Cardioprotection with adenosine-regulating agent, GP531: effects on no-reflow, infarct size, and blood flow following ischemia/ reperfusion in the rabbit. J Cardiovasc Pharmacol Ther 2010; 15: 60-7.

[19] Pifarre R, Cox WD, Jasuja M, Neville WE. Helium in the prevention of ventricular fibrillation. Dis Chest 1969; 56: 135-8.

[20] Heinen A, Huhn R, Smeele KM, et al. Helium-induced preconditioning in young and old rat heart: impact of mitochondrial $\mathrm{Ca}(2+)$-sensitive potassium channel activation. Anesthesiology 2008; 109: 830-6.

[21] Huhn R, Weber NC, Preckel B, et al. Age-related loss of cardiac preconditioning: impact of protein kinase A. Exp Gerontol 2012; 47: 116-21.

[22] Lucchinetti E, Wacker J, Maurer C, et al. Helium breathing provides modest antiinflammatory, but no endothelial protection against ischemia-reperfusion injury in humans in vivo. Anesth Analg 2009; 109: 101-8.

[23] Fukuda K, Asoh S, Ishikawa M, Yamamoto Y, Ohsawa I, Ohta S. Inhalation of hydrogen gas suppresses hepatic injury caused by ischemia/reperfusion through reducing oxidative stress. Biochem Biophys Res Commun 2007; 361: 670-4.

(C) Hale et al.; Licensee Bentham Open.

This is an open access article licensed under the terms of the Creative Commons Attribution Non-Commercial License (http://creativecommons.org/licenses/ by-nc/3.0/) which permits unrestricted, non-commercial use, distribution and reproduction in any medium, provided the work is properly cited. 